\title{
High-Performance Low-Temperature Polycrystalline-Silicon Thin Film Transistors with Submicron-Dot-Array Doped Active Channel
}

\author{
Meng Zhang, Wei Zhou, Rongsheng Chen, Shuming Chen, Man Wong and Hoi-Sing Kwok* \\ Center for Display Research and Department of Electronic and Computer Engineering \\ The Hong Kong University of Science and Technology, Hong Kong
}

\begin{abstract}
A novel method named as submicron-dot-array (SDA) doping is developed for the fabrication of low-temperature polycrystallinesilicon thin film transistors (TFTs). All electrical parameters are improved by employing SDA structure. It is worth mentioning that the mobility of fabricated device is 4 times of conventional TFTs. The proposed SDA method has great potential for system-onpanel applications.
\end{abstract}

Keywords: Low-temperature polycrystalline silicon, thin film transistors, submicron-dot-array doping

\section{Introduction}

Low-temperature polycrystalline-silicon (LTPS) technologies are actively being developed to generate high performance thin film transistors (TFTs) for system-on-panel applications [1]. Highperformance LTPS TFTs with low operating voltage, steep subthreshold swing $(S S)$ and large on-state-current/off-statecurrent $\left(I_{o n} / I_{o f f}\right)$ ratio are desired to accomplish the above purpose. However, polycrystalline-silicon (poly-Si) thin film has numerous grain boundaries (GBs) inside [2-4], resulting in small field-effect mobility $\left(\mu_{F E}\right)$, poor $S S$ and small $I_{o n} / I_{o f f}$ ratio in poly-Si TFTs and thus seriously limiting its application into driving circuits of active matrix displays. Several methods [5-7] have been proposed to reduce the GBs, such as high temperature annealing [5], plasma passivation [6] and bridged-grain (BG) technique [7]. For the high temperature annealing [5], the reported temperature often exceeds $700^{\circ} \mathrm{C}$, which is incompatible to LTPS technology. For the plasma passivation, it often brings process variation and reliability issues [6]. As to the BG technique [7], the BG lines are one dimensional and thus the device in the layout must be put in the specific position, which would sacrifice the circuit area and increase the difficulty for circuit designers. All methods above have critical shortcomings.

In this work, a new method named as submicron-dot-array (SDA) doping is proposed to improve device performance. The proposed SDA doping method is low temperature compatible, two dimensional and without process variation and reliability issues. With the SDA doping in the active channel, device shows great improvement in terms of $\mu_{F E}, S S$ and $I_{o n} / I_{o f f}$ ratio. It is worth mentioning that the $\mu_{F E}$ of the proposed TFTs with SDA doped channel is up to $58.4 \mathrm{~cm}^{2} / \mathrm{Vs}$, which is 3 times larger than that of conventional poly-Si TFTs. The test results suggest that the proposed SDA method is suitable for system-on-panel application.

\section{Experimental}

The cross-sectional illustration of device fabrication process flow is shown in Fig.1. First, 500-nm-thick thermal oxide was grown on 4-inch c-Si wafers in furnace. Then, $100 \mathrm{~nm}$ a-Si active layer was deposited by low-pressure chemical vapor deposition. Solidphase-crystallized (SPC) process was then carried out at $600^{\circ} \mathrm{C}$ for 24 hours in $N_{2}$ ambient. After SPC crystallization, the photoresist
(PR) was coated on the top of poly-Si thin film and then patterned into SDA structure through laser interference lithography as shown in the Figure 1a. The scanning electron microscope (SEM) image and atomic force microscope (AFM) image of SDA patterns after lithography are shown in Figure $2 a$ and Figure $2 b$ respectively. The diameter $(d)$ of submicron dot is $0.5 \mu \mathrm{m}$. Boron ions were then implanted into the exposed areas of the SPC ploySi film, as shown in the Figure 1a. After implantation, the PR was removed and the SDA doping pattern is formed as shown in Figure $1 \mathrm{~b}$. Then active islands were patterned, followed by $100 \mathrm{~nm}$ low temperature oxide (LTO) deposition as gate dielectric. Then $300 \mathrm{~nm}$ aluminum was sputtered and patterned as gate electrode. Next, self-aligned $35 \mathrm{keV}$ boron implantation was done at a dosage of $4 \times 10^{15} \mathrm{~cm}^{-2}$. 500nm LTO was then deposited and contact holes were defined. $700 \mathrm{~nm} \mathrm{Al-1 \% Si} \mathrm{was} \mathrm{sputtered.} \mathrm{After}$ patterning the metal layer, the devices were sintered in forming gas for $30 \mathrm{~min}$ at $420^{\circ} \mathrm{C}$. No further passivation was applied to these devices. The cross-sectional of device structure is shown in Figure 1c. The fabricated TFT with SDA doped channel is named SDA TFT. For the control wafer, no SDA doping is performed.

For characterizations, the Agilent $4156 \mathrm{C}$ semiconductor parameter analyzer is used to test the devices' transfer and output curves. The $\mu_{F E}$ is extracted from expression,

$$
\mu_{F E}=\frac{L d_{o x} G_{m}}{W \varepsilon_{o x} V_{d s}}
$$

where $d_{o x}, \varepsilon_{o x}$ and $G_{m}$ are physical gate dielectric thickness, gate dielectric permittivity and maximum of transconductance at $V_{d s}=-5 \mathrm{~V}$. The $S S$ is defined by the slope of $\log \left|I_{d}\right|$ in $10^{-10} \mathrm{~A}$ range at $V_{d s}=-5 \mathrm{~V}$. The $I_{\text {on }} / I_{\text {off }}$ ratio equals to maximum current over minimum current within the measure range at $V_{d s}=-5 \mathrm{~V}$. All TFTs used in this study have width $(W) /$ length $(L)=10 / 10 \mu \mathrm{m}$.
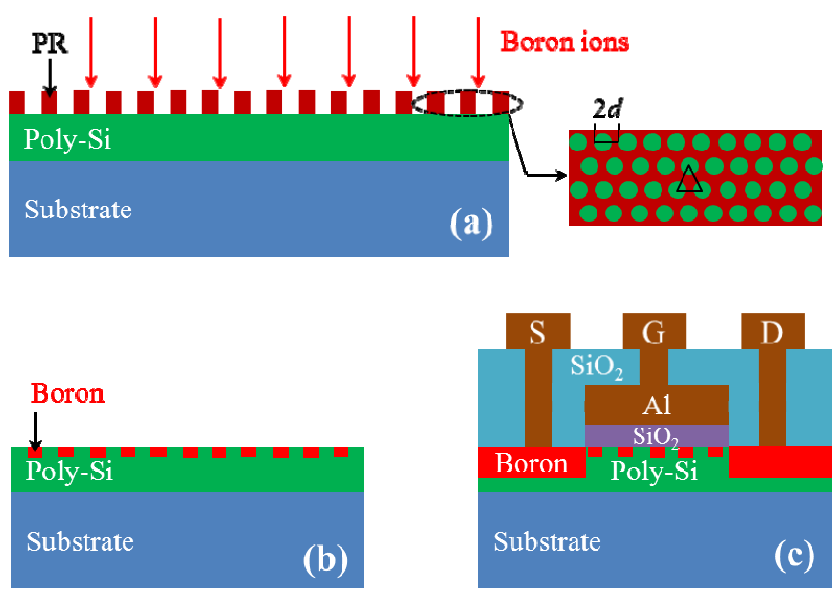

Figure 1: (a) The cross-sectional schematic and top view of SDA pattern formation. (b) The cross-sectional schematic of active channel with SDA boron doping. (c) The crosssectional of device structure. 


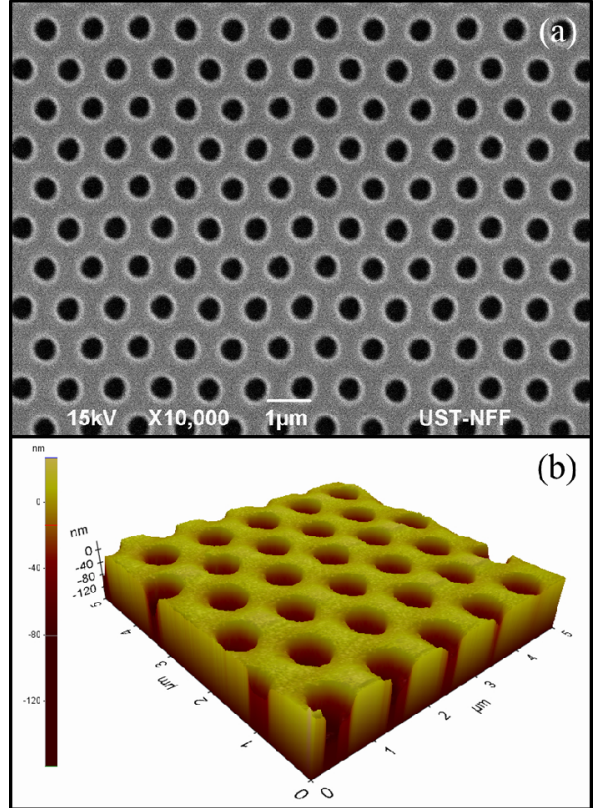

Figure 2: (a) SEM image and (b) AFM image of SDA pattern after lithography.

\section{Results and Discussion}

The transfer characteristics of SDA TFT and control TFT are shown in Figure 3. The inset are transfer curves plotted in the linear scale, measured at $V_{d s}=-0.1 \mathrm{~V}$. Compared to the control TFT, SDA TFT obviously show better performance in terms of higher $I_{o n}$, steeper $S S$ and lower gate-induced drain leakage (GIDL) current [8]. The $\mu_{F E}$ increases to $58.4 \mathrm{~cm}^{2} / \mathrm{Vs}$ for SDA TFT with $d=0.5 \mu \mathrm{m}$, which is 4 times of control TFT without SDA doping. The measured data and the extracted device parameters are summarized in Table I.

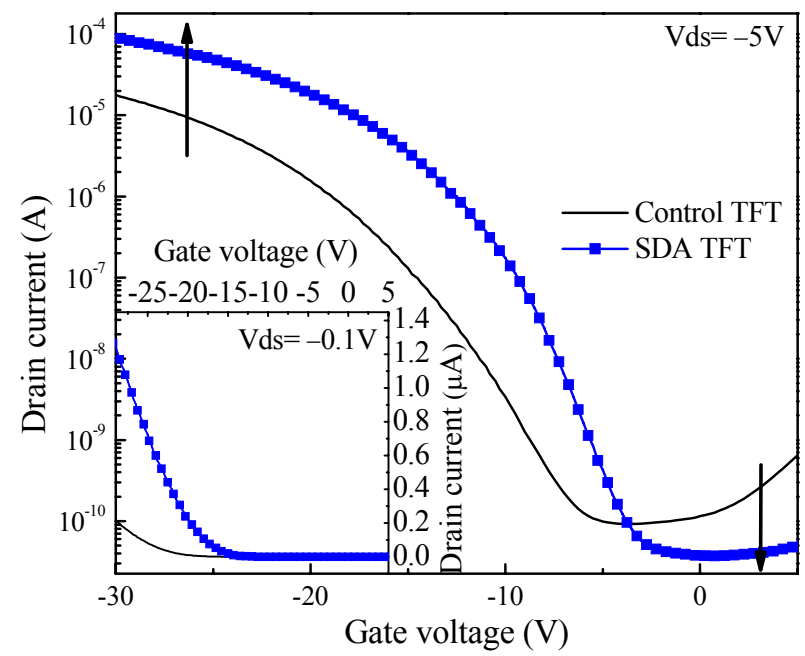

Figure 3: Transfer curves of SDA TFT and control TFT plotted in logarithmic scale, measured at $V_{d s}=-5 \mathrm{~V}$. The inset are transfer curves in the linear scale, measured at $V_{d s}=-0.1 \mathrm{~V}$.

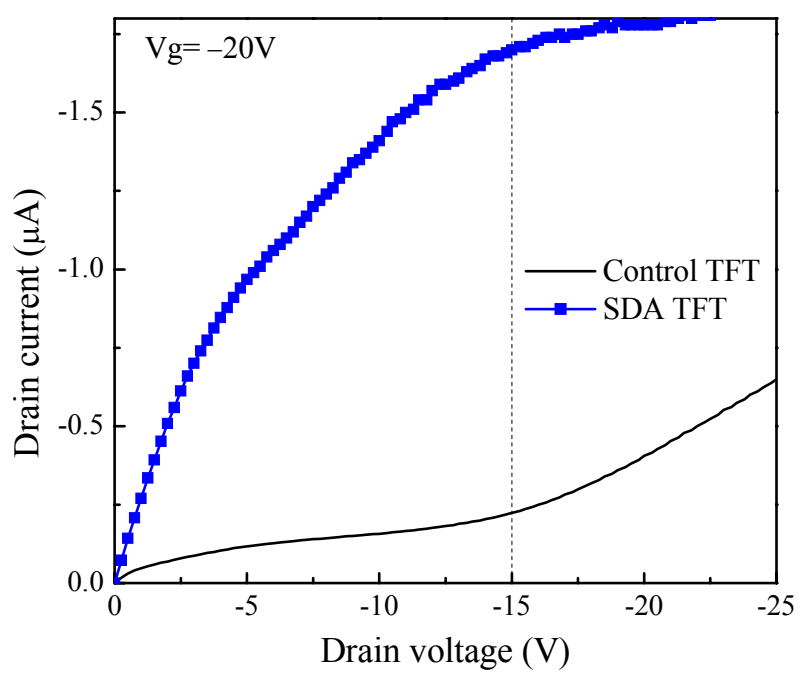

Figure 4: Output curves of SDA TFT and control TFT measured at $V_{g}=-20 \mathrm{~V}$.

Table I

Device parameters of SDA TFTs and control TFTs

\begin{tabular}{cccc}
\hline & $\begin{array}{c}\boldsymbol{\mu}_{\boldsymbol{F E}} \\
\left(\mathbf{c m}^{2} / \boldsymbol{V} \boldsymbol{s}\right)\end{array}$ & $\begin{array}{c}\boldsymbol{S S} \\
(\boldsymbol{V} / \mathbf{d e c})\end{array}$ & $\begin{array}{c}\boldsymbol{I}_{\text {on }} / \mathbf{I}_{\text {off }} \\
\left(\times \mathbf{1 0}^{\mathbf{5}}\right)\end{array}$ \\
\hline Control TFTs & 14.6 & 1.49 & 5.09 \\
SDA TFTs & 58.4 & 1.02 & 51.94 \\
\hline
\end{tabular}

Shown in the Figure 4 are output curves of SDA TFT and control TFT measured at $\mathrm{Vg}=-20 \mathrm{~V}$. Consistent with transfer curves shown in Fig.4, SDA TFT exhibits lager on current. Besides enhancing on current, it can be also observed that the kink current can be greatly suppressed by employing SDA structures, indicating improved hot carrier reliability [7-8].

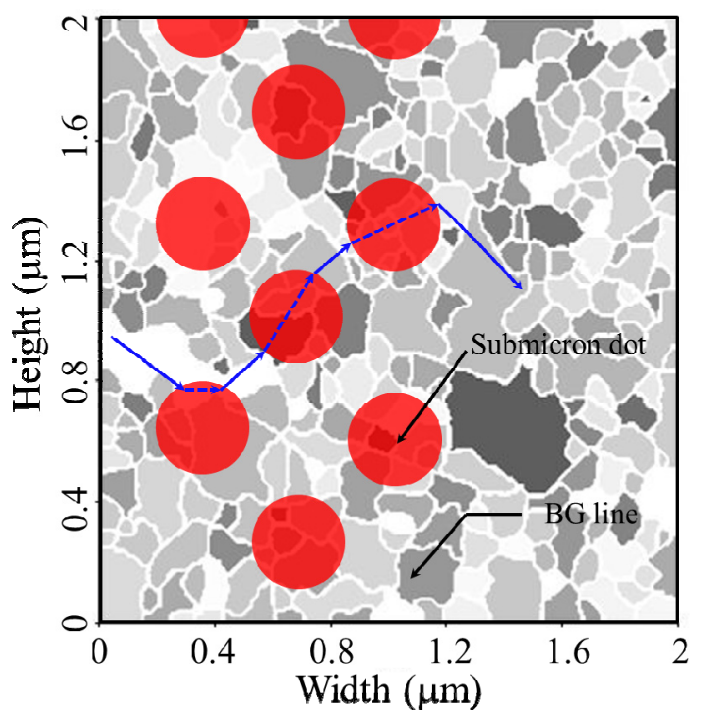

Figure 5: Schematic of current flow in poly-Si thin film with SDA doping based on grain detection result. 
To understand the conduction mechanism of SDA TFTs, the SPC poly-Si film is first treated by tetramethylammonium hydroxide etching. Then AFM is applied to confirm the grain size through examining the surface topography. With the help of the AFM watershed grain detection program [9], it was found that the mean value of the grain size is about $150 \mathrm{~nm}$, as shown in Figure 5. These small size grains result in high GB density. When the carries transfer from drain to source, they will surmount lots of barriers at GBs and thus limiting the on current. As discussed in ref. [7], when the carrier flows, it will pick the most direct path with less GBs. Once the carrier enters into doped region, it can pick any path freely since in the heavily doped region no barriers are supposed to be existed. In Figure 5, the red dots represent for heavily doped SDA. The principle of SDA is to build bridges to link the small grains and enhance on current, as indicated by blue arrows in Figure 5. For the off state, the GIDL current increases exponentially as a function of this reverse electric field [8]. The SDA structure can effectively terminate the electric field, resulting in lower GIDL current, compared to the control TFT.

\section{Conclusion}

In this work, the SPC poly-Si TFTs using SDA doped channel is first demonstrated. By employing SDA method, the device performance is greatly enhanced, especially for improvement of $\mu_{F F}$. The conduction mechanism of SDA TFTs is analyzed and discussed.

\section{Acknowledgements}

Authors would like to thank Prof. Mingxiang Wang in Soochow University for valuable discussions. This work was supported by Hong Kong Government Research Grants Council Theme-Based Research Scheme under Grant T23-713/11-1.

\section{Reference}

[1] M. Zhang, M. Wang, H. Wang, and J. Zhou, "Degradation of Metal-Induced Laterally Crystallized n-Type Polycrystalline Silicon Thin-Film Transistors under Synchronized Voltage Stress," IEEE Trans. Electron devices, 2009, vol. 56, pp. 2726-2732.
[2] N. Yamauchi and R. Reif, "Polycrystalline Silicon Thin-Films Processed with Silicon Ion-Implantation and Subsequent Solid-Phase Crystallization - Theory, Experiments, and ThinFilm-Transistor Applications," 1994, J. Appl. Phys., vol. 75, pp. 3235-3257.

[3] M. Miyasaka and J. Stoemenos, "Excimer laser annealing of amorphous and solid-phase-crystallized silicon films," 1999 , J. Appl. Phys., vol. 86, pp. 5556-5565.

[4] Z. Meng, M. Wang and M. Wong, "High Performance Low Temperature Metal-Induced Unilaterally Crystallized Polycrystalline Silicon Thin Film Transistors for System-onPanel Applications," IEEE Trans. Electron devices, 2000, vol. 47, pp. 404-409.

[5] M. Wang, Z. Meng, and M. Wong, "The effects of high temperature annealing on metal-induced laterally crystallized polycrystalline silicon," IEEE Trans. Electron Devices, 2000 vol. 47, no. 11, pp. 2061-2067.

[6] H. C. Cheng, F. S. Wang, and C. Y. Huang, "Effects of NH3 plasma passivation on N-channel polycrystalline silicon thinfilm transistors," IEEE Trans. Electron Devices, 1997, vol. 44, no. 1, pp. 64-68.

[7] W. Zhou, Z. Meng, S. Zhao, M. Zhang, R. Chen, M. Wong and H. S. Kwok, "Bridged-Grain Solid-Phase-Crystallized Polycrystalline-Silicon Thin-Film Transistors", IEEE Electron Device Lett., 2012, vol. 33, pp. 1414-1416.

[8] M. Zhang, M. Wang, X. Lu, M. Wong and H. S. Kwok, "Analysis of Degradation Mechanisms in Low-Temperature Polycrystalline Silicon Thin-Film Transistors under Dynamic Drain Stress", IEEE Trans. Electron devices, 2012, vol. 59, pp.1730-1737.

[9] J. Barraud, "The use of watershed segmentation and GIS software for textural analysis of thin sections," J. Volcanol. Geotherm. Res, 2006, vol. 154, pp. 17-33 